\title{
On determinantal recurrence relations of banded matrices
}

\author{
Zhibin $\mathrm{Du}^{1}$, Carlos M. da Fonseca ${ }^{2, *}$, António Pereira ${ }^{3}$ \\ ${ }^{1}$ School of Software, South China Normal University, Foshan, Guangdong 528225, China \\ ${ }^{2}$ Kuwait College of Science and Technology, Doha District, Safat 13133, Kuwait \\ ${ }^{3}$ Dept. of Mathematics, University of Aveiro, 3810-193 Aveiro, Portugal \\ *Corresponding author: c.dafonseca@kcst.edu.kw
}

\begin{abstract}
We provide an algorithm based on a less-known result about recurrence relations for the determinants of banded matrices. As a consequence, we prove recent conjectures on the determinants of particular classes of pentadiagonal matrices and simple alternative proofs for other results.
\end{abstract}

Keywords: Algorithm, banded matrices, determinant, pentadiagonal matrices

2000 Mathematics Subject Classification:15A18, 65F15, 15B05, 42C05

\section{Introduction}

The standard tridiagonal matrices are square matrices of the form

$$
\left(\begin{array}{ccccc}
* & * & & & \\
* & * & * & & \\
& * & \ddots & \ddots & \\
& & \ddots & \ddots & * \\
& & & * & *
\end{array}\right)
$$

where the non-mentioned entries should be read as zero. Analogously, we have the pentadiagonal matrices which are of the form

$$
\left(\begin{array}{ccccccc}
* & * & * & & & & \\
* & * & * & * & & & \\
* & * & \ddots & \ddots & \ddots & & \\
& * & \ddots & \ddots & \ddots & \ddots & \\
& & \ddots & \ddots & \ddots & \ddots & * \\
& & & \ddots & \ddots & \ddots & * \\
& & & & * & * & *
\end{array}\right) .
$$

These matrices (particularly, those which are Toeplitz) occur in many numerical problems involving different kinds of differential equations and they also naturally emerge in many areas of pure, applied, and numerical mathematics, engineering, statistics, signal processing, among many others, with particular emphasis to the computational problems related to the calculation of spectra, determinant, permanent, characteristic polynomial, inverse, power, and different types of decompositions (Arbenz, 1991; Arıkan \& Kılıc, 2017; Cinkir, 2012; Diele \& Lopez, 1998; Elouafi, 2013; Elouafi, 2011; Hadj \& Elouafi, 2008; Jia et al., 2016; Kratz, 2010; Kratz \& Tentler, 2008; K1lıc \& El-Mikkawy, 2008; Montaner \& 
Alfaro, 1995; Marr \& Vineyard, 1988; Sweet, 1969). There are many extensions of these matrices which deserved attention in many areas of research as we can find, for example, in (Anđelić \& da Fonseca, 2021; Arbenz, 1991; Egerváry \& Szász, 1928; da Fonseca \& Y1lmaz, 2015; Kratz, 2010; Kratz \& Tentler, 2008; Losonczi, 1992; McMillen, 2009; Ohashi et al., 2015; Takahira et al., 2019). All of them belong to the family of the banded matrices.

Definition 1.1. Let $r$ and $s$ be nonnegative integers. A square matrix $A=\left(a_{i, j}\right)_{i, j \geqslant 0}$ is called an $(r, s)$ banded matrix if $a_{i, j}=0$ unless $-s \leqslant i-j \leqslant r$.

The bandwidth of an $(r, s)$-banded matrix is $r+s+1$. For example, any tridiagonal matrix of the form (1) is a (1,1)-banded matrix, while the pentadiagonal matrix (2) is (2,2)-banded, with bandwidths, respectively 3 and 5 . Another example is an $n \times n$ Hessenberg matrix. Here the bandwidth is $n+1$.

Since we are mainly interested on the discussion of the determinants of certain banded matrices, it is useful to recall the next theorem.

Theorem 1.1. (Zakrajšek \& Petkovšek, 2004, Theorem 2) Let $A=\left(a_{i, j}\right)_{i, j \geqslant 0}$ be an $(r, s)$-banded matrix and $\delta=\left(\begin{array}{c}r+s \\ r\end{array}\right)$. Denote by $d_{n}$ the principal minor of $A$ consisting of the entries indexed by the rows and columns $0,1, \ldots, n$. Then, for $n \geqslant \delta$, the sequence $\left(d_{n}\right)_{n \geqslant 0}$ satisfies a nontrivial homogeneous linear recurrence of the form

$$
d_{n}=\sum_{n=1}^{\delta} R_{k} d_{n-k},
$$

where each $R_{k}$ is a homogeneous rational function of degree $k$ of entries $a_{n-i, n-j}$, with $0 \leqslant i \leqslant \delta-1$ and $-s \leqslant j \leqslant r+\delta-1$.

Recall that a rational function $f$ is homogeneous of degree $k$, if $f\left(t x_{1}, \ldots, t x_{n}\right)=t^{k} f\left(x_{1}, \ldots, x_{n}\right)$.

This theorem and its proof are largely unknown, for example, in the linear algebra community, and both can be quite useful in many instances.

In this note, we describe a Mathematica (Wolfram Research, Inc., 2020) algorithm based on Theorem 1.1 which allows us to provide alternative proofs to some recent results and answers to two conjectures.

\section{The algorithm}

In this section we provide the implementation of the main Mathematica functions, based on Theorem 1.1 , that allowed us to compute the results contained in the following sections.

In the listing below, the function equations constructs the $\delta=\left(\begin{array}{c}r+s \\ r\end{array}\right)$ equations associated with the determinants of a general $(r, s)$-banded matrix with elements $\mathbf{a}[\mathbf{i}, \mathbf{j}]$. These equations are used by function $f$ indRecurrenceEquation to compute the corresponding recurrence relation

$$
\mathrm{d}[1, \mathrm{n}]==\alpha_{1} \mathrm{~d}[1, \mathrm{n}-1]+\cdots+\alpha_{\delta} \mathrm{d}[1, \mathrm{n}-\delta] .
$$

\section{Listing 1. Main functions}

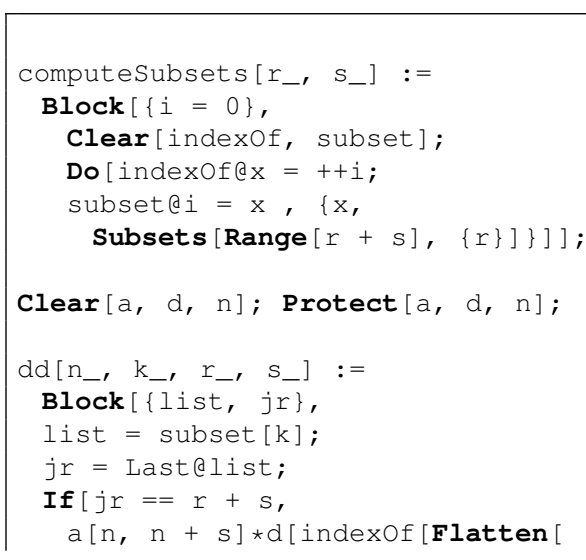




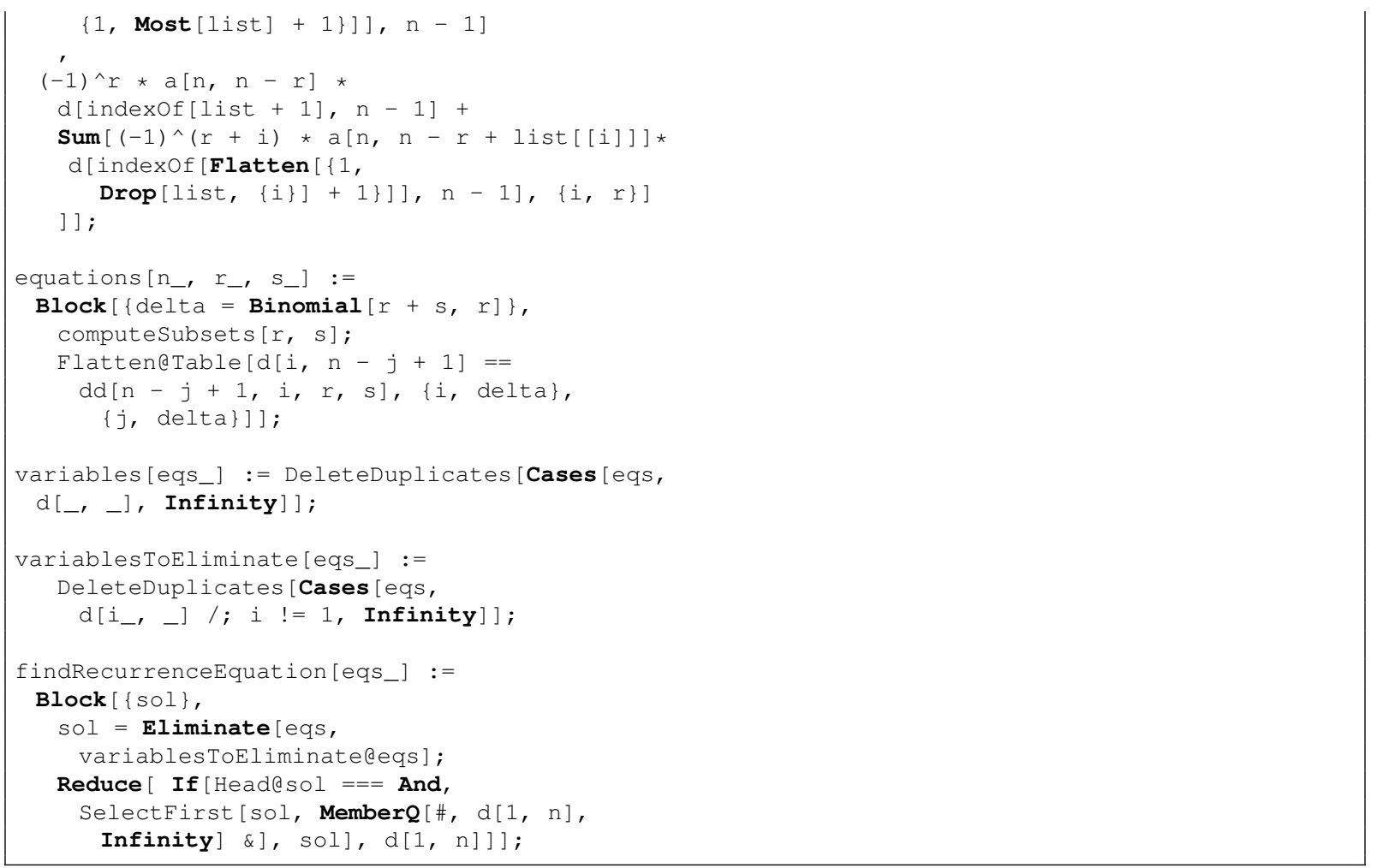

Some remarks are in order. First the number of equations necessary to compute the recurrence relation for the determinants of an $(r, s)$-banded matrix increases very rapidly with the bandwidth $r+s+1$ of the matrix. Thus, the process of variable elimination used by function $\mathrm{f}$ indRecurrence Equation can be quite time-consuming. Also, for a general banded matrix, the coefficients $\alpha_{i}$ in the recurrence relation can be large and complex expressions.

Given a particular banded matrix with concrete elements $b[i, j]$, it is better to start by transforming the general equations into the particular ones before computing the recurrence relation:

eqs $=$ equations $[n, r, s] / \cdot\left\{a\left[i_{-}, j \_\right]:>b[i, j]\right\}$;

findRecurrenceEquation [eqs];

Mathematica's Eliminate function, used in findRecurrenceEquation, can be very slow when applied to equations computed for general matrices containing several parameters. One way to overcome this problem is by first computing the matrix associated with the linear system with a clever ordering of the variables and then perform row reduction of this matrix. An implementation of this method is next listed.

Listing 2. An improved recurrence finder

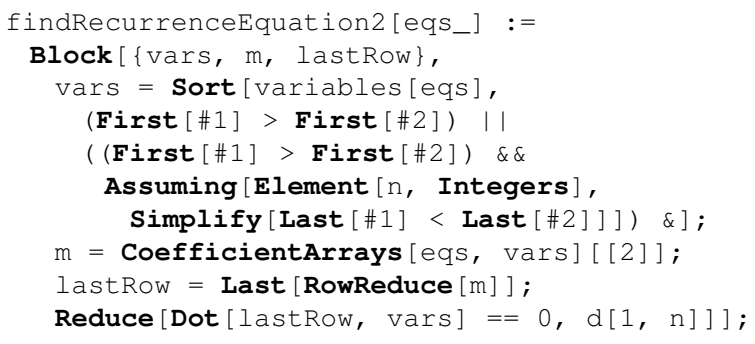

Finally, an explicit formula for the determinants of a banded matrix can be obtained from the computed recurrence relation by using Mathematica's function RSolve.

In the next sections we will find some applications of this algorithm. 


\section{Recurrence relations}

For more than five decades there has been an effort for investigating the structure of the determinant of pentadiagonal matrices in order to develop efficient methods for determining they spectra. We believe that R.A. Sweet first determined in 1969 a recursive formula for the determinant of any matrix of the form (2) and, consequently, a method to determine explicitly and locate the eigenvalues of such matrices (Sweet, 1969). For Toeplitz matrices

$$
P_{n}=\left(\begin{array}{ccccccc}
a & b & c & & & & \\
d & a & b & c & & & \\
e & d & \ddots & \ddots & \ddots & & \\
& e & \ddots & \ddots & \ddots & \ddots & \\
& & \ddots & \ddots & \ddots & \ddots & c \\
& & & \ddots & \ddots & \ddots & b \\
& & & & e & d & a
\end{array}\right)_{n \times n}
$$

Sweet's 7-term recurrence relation (Sweet, 1969, (10)) reads as

$p_{k}=a p_{k-1}+(e c-b d) p_{k-2}+\left(c d^{2}+b^{2} e-2 a c e\right) p_{k-3}+c e(c e-b d) p_{k-4}+a(c e)^{2} p_{k-5}-(c e)^{3} p_{k-6}$,

for $k \geqslant 5$, with initial conditions $p_{-1}=0, p_{0}=1$ and $p_{k}=\operatorname{det} P_{k}$, for $k=1,2,3,4$. This recurrence relation has been re-discovered in many instances, as in (Arbenz, 1991; Cinkir, 2012; Jia et al., 2016; Kratz \& Tentler, 2008; Montaner \& Alfaro, 1995), and was not always properly acknowledged.

We will provide the recent examples where we can find as an immediate application of the algorithm proposed in Section 2.

Recently, (Küçük \& Düz, 2017, Remark 9) claimed that the determinant of the 3-tridiagonal matrix

$$
T_{n}=\left(\begin{array}{ccccccc}
a & 0 & 0 & b & & & \\
0 & a & 0 & 0 & b & & \\
0 & 0 & \ddots & \ddots & \ddots & \ddots & \\
c & 0 & \ddots & \ddots & \ddots & \ddots & b \\
& c & \ddots & \ddots & \ddots & \ddots & 0 \\
& & \ddots & \ddots & \ddots & \ddots & 0 \\
& & & c & 0 & 0 & a
\end{array}\right)
$$

cannot be written in terms of a linear recurrence relation involving the determinants of its minors for $a=2 x, b=1$ and $c=-1$ (see also (da Fonseca, 2018b)). This is clearly wrong since, from Theorem 1.1 , with $r=s=3$, there is a recurrence relation $\delta+1=\left(\begin{array}{l}6 \\ 3\end{array}\right)+1=20+1$ terms for the $d_{n}$. Applying the algorithm, this recurrence is

$$
d_{n}=a d_{n-1}-b c d_{n-2}+a b c d_{n-3}-a^{2} b c d_{n-4}+a b^{2} c^{2} d_{n-5}-b^{3} c^{3} d_{n-6}+a b^{3} c^{3} d_{n-7}-b^{4} c^{4} d_{n-8}
$$

In matter of fact, due to the pattern of $T_{n}$ in this particular case, we may recover the exact formula for the recurrence relation established in (Trojovský \& Zvoníková, 2019), where the number of terms can be significantly reduced.

Theorem 3.1. Let $n>8$ be an integer and $d_{n}=\operatorname{det} T_{n}$, with $a=2 x, b=1$ and $c=-1$. Then the sequence $\left(d_{n}\right)_{n>8}$ satisfies the recurrence relation with 9 terms of the form

$$
d_{n}=2 x d_{n-1}-d_{n-2}+2 x d_{n-3}-4 x^{2} d_{n-4}+2 x d_{n-5}-d_{n-6}+2 x d_{n-7}-d_{n-8},
$$


with initial conditions

$$
\begin{aligned}
& d_{1}=2 x, d_{2}=4 x^{2}, d_{3}=8 x^{3} \\
& d_{4}=4 x^{2}\left(4 x^{2}-1\right), d_{5}=2 x\left(4 x^{2}-1\right)^{2} \\
& d_{6}=\left(4 x^{2}-1\right)^{3}, d_{7}=4 x\left(2 x^{2}-1\right)\left(4 x^{2}-1\right)^{2} \\
& d_{8}=(4 x)^{2}\left(2 x^{2}-1\right)^{2}\left(4 x^{2}-1\right) .
\end{aligned}
$$

From (da Fonseca \& Y1lmaz, 2015; Losonczi, 1992), one can deduce a compact formula for Theorem 3.1:

$$
d_{n}(x)=(\sqrt{b c})^{n} U_{q+1}^{r}\left(\frac{x-a}{2 \sqrt{b c}}\right) U_{q}^{3-r}\left(\frac{x-a}{2 \sqrt{b c}}\right),
$$

where

$$
n=3 q+r, \quad \text { with } r \in\{0,1,2\},
$$

and $\left\{U_{n}(x)\right\}_{n \geqslant 0}$ is the sequence of Chebyshev polynomials of the second kind.

Another approach can be found is in (da Silva, 2017) for the general case (3), where we can see that the formulas become quite intricate.

\section{Two conjectures}

Another recent and interesting problems were proposed in (Anđelić \& da Fonseca, 2021) involving the determinants of an extension to pentadiagonal matrices. It was conjectured the following.

Conjecture 1. The determinant of the matrix

$$
A_{n}=\left(\begin{array}{ccccccc}
1 & 1 & & & & & 1 \\
1 & 1 & 1 & & & & \\
1 & 1 & \ddots & \ddots & & & \\
& 1 & \ddots & \ddots & \ddots & & \\
& & \ddots & \ddots & \ddots & \ddots & \\
& & & \ddots & \ddots & \ddots & 1 \\
& & & & 1 & 1 & 1
\end{array}\right)_{n \times n}
$$

is, for $n \geqslant 4$,

$$
\operatorname{det} A_{n}=\left\{\begin{array}{ccc}
1 & \text { if } n \equiv 0 & (\bmod 4) \\
2 & \text { if } n \equiv 1 & (\bmod 4) \\
-1 & \text { if } n \equiv 2 & (\bmod 4) \\
0 & \text { if } n \equiv 3 & (\bmod 4)
\end{array}\right.
$$

Conjecture 2. The determinant of the matrix

$$
B_{n}=\left(\begin{array}{ccccccc}
1 & 1 & & & & 1 & \\
1 & 1 & 1 & & & & 1 \\
1 & 1 & \ddots & \ddots & & & \\
& 1 & \ddots & \ddots & \ddots & & \\
& & \ddots & \ddots & \ddots & \ddots & \\
& & & \ddots & \ddots & \ddots & 1 \\
& & & & 1 & 1 & 1
\end{array}\right)_{n \times n}
$$


is, for $n \geqslant 4$,

$$
\operatorname{det} B_{n}=\left\{\begin{array}{ccc}
0 & \text { if } n \equiv 0 & (\bmod 4) \\
2 & \text { if } n \equiv 1 & (\bmod 4) \\
3 & \text { if } n \equiv 2 & (\bmod 4) \\
1 & \text { if } n \equiv 3 & (\bmod 4)
\end{array} .\right.
$$

Both conjectures have recently attracted the attention of many researchers (Amanbek et al., 2020; Kurmanbek et al., 2020; Shitov, 2021).

Using the algorithm developed in Section 2, we are able here to confirm these conjectures by providing a compact formula for both. First we prove an auxiliary result.

Lemma 4.1. Let $F_{n}$ be the all 1's (2,1)-banded matrix of order $n$, that is, defined by $f_{i, j}=1$ if $-1 \leq$ $i-j \leq 2$, and 0 elsewhere. Then the sequence of determinants of $F_{n}$ satisfies the recurrence relation

$$
d_{n}=d_{n-4}
$$

with initial conditions $d_{1}=d_{4}=1$ and $d_{2}=d_{3}=0$. Moreover, for $n \geq 1$, this recurrence has the explicit solution

$$
d_{n}=\frac{1}{2}\left(\sin \left(\frac{n \pi}{2}\right)+\cos \left(\frac{n \pi}{2}\right)+1\right) .
$$

Proof. Equation (4) was obtained by using the algorithm presented in Section 2. The explicit solution was obtained by using Mathematica's Rsolve function.

Next we are able to prove both conjectures.

Theorem 4.2. The sequence of determinants of $A_{n},\left(d_{n}\right)_{n \geq 3}$, satisfies explicitly

$$
d_{n}=\frac{1}{2}\left(1-(-1)^{n}\right)+\sin \left(\frac{n \pi}{2}\right)+\cos \left(\frac{n \pi}{2}\right) .
$$

Proof. Developing the determinant of $A_{n}$ from its first row, we get

$$
\operatorname{det} A_{n}=\operatorname{det} A_{n}^{(1,1)}-\operatorname{det} A_{n}^{(1,2)}+(-1)^{n+1} \operatorname{det} A_{n}^{(1, n)} .
$$

$A_{n}^{(1,1)}$ is a full, all 1's, order $n-1,(2,1)$-banded matrix. From Lemma 4.1, the general recurrence relation for the determinant of an order $n$ matrix of this kind is (4). Thus the associated characteristic polynomial is $p_{1}(x)=x^{4}-1$.

The matrix $A_{n}^{(1,2)}$ is a $(2,1)$-banded matrix of order $n-1$ where $a_{3,1}=0$ and $a_{i, j}=1$ when $-1 \leq i-j \leq 2$ and $(i, j) \neq(3,1)$. By using the algorithm presented in Section 2, we conclude that the general recurrence relation for the determinant of an order $n$ matrix of this kind is also (4). Therefore, the associated characteristic polynomial is $p_{2}(x)=p_{1}(x)$.

Similarly, $A_{n}^{(1, n)}$ is a $(1,2)$-banded matrix of order $n-1$ where $a_{i, j}=1$ when $-2 \leq i-j \leq$ 1. Noticing that $A_{n}^{(1, n)}$ is the transpose of $A_{n}^{(1,1)}$, we get that the general recurrence relation for the determinant of an order $n$ matrix of this kind is also (4).

Now, notice that the recurrence relation for the sequence $d_{n}^{\prime}=(-1)^{n} d_{n}$, where $d_{n}$ is given by (4), is also $d_{n}^{\prime}=d_{n-4}^{\prime}$ with associated characteristic polynomial given by $p_{3}(x)=p_{1}(x)$.

We can now conclude that the characteristic polynomial associated to the recurrence $d_{n}=\operatorname{det} A_{n}$ is given by $\operatorname{lcm}\left(p_{1}(x), p_{2}(x), p_{3}(x)\right)=x^{4}-1$, hence $d_{n}=d_{n-4}$ for $n>4$.

Finally, the explicit solution was obtained by using Mathematica's function.

Corollary 4.3. The sequence of determinants of $A_{n},\left(d_{n}\right)_{n \geq 1}$, satisfies the recurrence relation (4), with initial conditions $d_{1}=2, d_{2}=-1, d_{3}=0$, and $d_{4}=1$. 
Theorem 4.4. The sequence of determinants of $B_{n},\left(d_{n}\right)_{n \geq 4}$, satisfies the recurrence relation (4), with initial conditions $d_{1}=2, d_{2}=3, d_{3}=1$, and $d_{4}=0$. Moreover, for $n \geq 1$, this recurrence has the explicit solution

$$
\operatorname{det} B_{n}=\frac{1}{2}\left(\sin \left(\frac{n \pi}{2}\right)-3 \cos \left(\frac{n \pi}{2}\right)+3\right) .
$$

Proof. Let us start expanding the determinant of $B_{n}$ along the first row, i.e.,

$$
\operatorname{det} B_{n}=\operatorname{det} B_{n}^{(1,1)}-\operatorname{det} B_{n}^{(1,2)}+(-1)^{n} \operatorname{det} B_{n}^{(1, n-1)} .
$$

Notice that $B_{n}^{(1,1)}$ is equal to matrix $A_{n-1}$ in the previous theorem. Thus the recurrence relation for the determinants of $B_{n}^{(1,1)}$ is (4).

Next, take $C_{n-1}=B_{n}^{(1,2)}$ and expand the determinant of $C_{n-1}$ along the first row:

$$
\operatorname{det} C_{n-1}=\operatorname{det} C_{n-1}^{(1,1)}-\operatorname{det} C_{n-1}^{(1,2)}+(-1)^{n} \operatorname{det} C_{n-1}^{(1, n-1)} .
$$

The matrix $C_{n-1}^{(1,1)}$ is of the same kind as matrix $A_{n}^{(1,1)}$ in the proof of Theorem 4.2 (a $(2,1)$-banded matrix of order $n-2$ ). Hence it also satisfies the recurrence (4). On the other hand, the matrix $C_{n-1}^{(1,2)}$ is changed into $(2,1)$-banded of order $n-3$ after expanding along the first column. The general recurrence relation for the determinant of this kind of matrix is again (4). The matrix $C_{n-1}^{(1, n-1)}$ becomes $(1,2)$-banded of order $n-3$ after expanding along the first column. The determinants of these matrices also satisfy the general recurrence relation (4).

It is now simple to conclude that the determinants of $B_{n}^{(1,2)}$ also satisfy (4).

Next, we consider $E_{n-1}=B_{n}^{(1, n-1)}$ and expand the determinant of $E_{n-1}$ along the last column. We get

$$
\operatorname{det} E_{n-1}=(-1)^{n} \operatorname{det} E_{n-1}^{(1, n-1)}-\operatorname{det} E_{n-1}^{(n-2, n-1)}+\operatorname{det} E_{n-1}^{(n-1, n-1)} .
$$

Now, $E_{n-1}^{(1, n-1)}$ is an upper triangular matrix with main diagonal all equal to 1 . Expanding the determinant of $E_{n-1}^{(n-2, n-1)}$ along the last row, we obtain a full $(1,2)$-banded matrix of order $n-3$ with banded elements all equal to 1 . Therefore, from Lemma 4.1, the sequence of determinants satisfies (4). The matrix $E_{n-1}^{(n-1, n-1)}$ is also a full $(1,2)$-banded matrix of order $n-2$ with banded elements all equal to 1 . Thus the sequence of determinants satisfies (4). It is now simple to conclude that the sequence of determinants for $E_{n-1}$ also satisfies (4).

Recalling (6) we conclude that the sequence of determinants of $B_{n}$ satisfies $d_{n}=d_{n-4}$.

Corollary 4.5. The sequence of determinants of $B_{n},\left(d_{n}\right)_{n \geq 1}$, satisfies the recurrence relation (4), with initial conditions $d_{1}=2, d_{2}=3, d_{3}=1$, and $d_{4}=0$.

From both corollaries, we guess that the recurrence relation (4) is indeed satisfied for a larger (even much larger) family of pentadiagonal matrices. We leave this as an open research problem.

For other related recurrences on other families of matrices, the reader is referred, for example, to (da Fonseca, 2018a).

\section{Acknowledgements}

The authors are indebted to the referees for the valuable comments.

The project was funded by Kuwait Foundation for the Advancement of Sciences under project code: PR19-16SM-04. 


\section{References}

Amanbek, Y et al. (2020). Explicit determinantal formula for a class of banded matrices, Open Math. $18,1227-1229$.

An delï, M. \& da Fonseca, C.M. (2021). Some determinantal considerations for pentadiagonalmatrices, Linear Multilinear Algebra, DOI: 10.1080/03081087.2019.1708845

Arbenz, P. (1991). Computing eigenvalues of banded symmetric Toeplitz matrices, SIAM J. Sci. Stat. Comput. 12, 743-754.

Arıkan, T. \& Kılıc (2017). A class of non-symmetric band determinants with the Gaussian $q$-binomial coefficients, Quaest. Math. 40, 645-660.

Cinkir, Z. (2012). An elementary algorithm for computing the determinant of pentadiagonal Toeplitz matrices, J. Comput. Appl. Math. 236, 2298-2305.

Diele, F. \& Lopez, L. (1998). The use of the factorization of five-diagonal matrices by tridiagonal Toeplitz matrices, Appl. Math. Lett. 11, 61-69.

Egerváry, E. \& Szász, O. (1928). Einige Extremalprobleme im Bereiche der trigonometrischen Polynome, Math. Z. 27, 641-652.

Elouafi, M. (2011). An explicit formula for the determinant of a skew-symmetric pentadiagonal Toeplitz matrix, Appl. Math. Comput. 218, 3466-3469.

Elouafi, M. (2013). A note for an explicit formula for the determinant of pentadiagonal and heptadiagonal symmetric Toeplitz matrices, Appl. Math. Comput. 435, 4789-4791.

da Fonseca, C.M. (2018b). On some conjectures regarding tridiagonal matrices, J. Appl. Math. Comput. Mech. 17, 13-17.

da Fonseca, C.M. (2018a). The eigenvalues of some anti-tridiagonal Hankel matrices, Kuwait J. Sci. $45,1-6$.

da Fonseca, C.M. \& Yllmaz, F. (2015). Some comments on $k$-tridiagonal matrices: Determinant, spectra, and inversion, Appl. Math. Comput. 270, 644-647.

Hadj, A.D.A. \& Elouafi, M. (2008). On the characteristic polynomial, eigenvectors and determinant of a pentadiagonal matrix, Appl. Math. Comput. 198, 634-642.

Jia, J., Yang, B. \& Li, S. (2016). On a homogeneous recurrence relation for the determinants of general pentadiagonal Toeplitz matrices, Comput. Math. Appl. 71, 1036-1044.

Kılıc, E. \&El-Mikkawy, M. (2008). A computational algorithm for special $n$ th-order pentadiagonal Toeplitz determinants, Appl. Math. Comput. 199, 820-822.

Kratz W. (2010). Banded matrices and discrete Sturm-Liouville eigenvalue problems, Adv. Difference Equ. 2009, Article ID 362627.

Kratz W. \& Tentler, M. (2008). Recursion formulae for the characteristic polynomial of symmetric banded matrices, Linear Algebra Appl. 428, 2482-2500.

Küçük, A.Z. \& Düz, M. (2017). Relationships between the permanents of a certain type of $k$-tridiagonal symmetric Toeplitz matrix and the Chebyshev polynomials, J. Appl. Math. Comput. Mech. 16, 75-86. 
Kurmanbek, B., Amanbek Y. \& Erlangga, Y. (2020). A proof of Anđelić-Fonseca conjectures on the determinant of some Toeplitz matrices and their generalization, Linear Multilinear Algebra, DOI: 10.1080/03081087.2020.1765959.

Losonczi, L. (1992). Eigenvalues and eigenvectors of some tridiagonal matrices, Acta Math. Hung. 60, 309-332.

Marr, R.B. \& Vineyard, G.H. (1988). Five-diagonal Toeplitz determinants and their relation to Chebyshev polynomials, SIAM J. Matrix Anal. Appl. 9, 579-586.

McMillen, T. (2009). On the eigenvalues of double band matrices, Linear Algebra Appl. 431, 1890-1897.

Montaner, J.M. \& Alfaro, M. (1995). On five-diagonal Toeplitz matrices and orthogonal polynomials on the unit circle, Numer. Algor. 10, 137-153.

Ohashi, A., Sogabe, T. \& Usuda, T.S. (2015). On decomposition of $k$-tridiagonal $\ell$-Toeplitz matrices and its applications, Spec. Matrices 7, 200-206.

Shitov, Y. (2021). The determinants of certain $(0,1)$ Toeplitz matrices, Linear Algebra App, to appear. da Silva, J.L. (2017). On the characteristic polynomial, eigenvectors and determinant of heptadiagonal matrices, Linear Multilinear Algebra 65, 1852-1866.

Sweet, R.A. (1969). A recursive relation for the determinant of a pentadiagonal matrix, Comm. ACM. 12, 330-332.

Takahira, S., Sogabe, T. \& Usuda, T.S. (2019). Bidiagonalization of $(k, k+1)$-tridiagonal matrices, Spec. Matrices 7, 20-26.

Trojovský, P. \& Zvoníková, I. (2019). On a recurrence for permanents of a sequence of 3 tridiagonalmatrices, J. Appl. Math. Comput. Mech. 18, 95-100.

Zakrajšek, H. \& Petkovšek, M. (2004). Pascal-like determinants are recursive, Adv. Appl. Math. 33, 431-450.

Wolfram Research, Inc. Mathematica, Version 12.2, Champaign, IL.

$\begin{array}{lr}\text { Submitted: } & 24 / 10 / 2020 \\ \text { Revised: } & 01 / 02 / 2021 \\ \text { Accepted: } & 02 / 02 / 2021 \\ \text { DOI: } \quad 10.48129 / \text { kjs.v49i1.11165 }\end{array}$

